\section{$\underset{\substack{\text { hommes } \\ \text { \& migrations }}}{ }$}

\section{Hommes \& migrations}

Revue française de référence sur les dynamiques

migratoires

1286-1287 | 2010

Les migrations subsahariennes

\title{
Un corps en exil
}

Entretien avec le danseur nigérian Qudus Onikeku

\section{Dagara Dakin et Qudus Onikeku}

Traducteur : Latifa Aït Naceur

\section{CpenEdition}

\section{Journals}

Édition électronique

URL : http://journals.openedition.org/hommesmigrations/1745

DOI : 10.4000/hommesmigrations. 1745

ISSN : 2262-3353

\section{Éditeur}

Musée national de l'histoire de l'immigration

Édition imprimée

Date de publication : 1 juillet 2010

Pagination : 174-177

ISSN : 1142-852X

Référence électronique

Dagara Dakin et Qudus Onikeku, « Un corps en exil », Hommes \& migrations [En ligne],

1286-1287 | 2010, mis en ligne le 29 mai 2013, consulté le 21 avril 2019. URL : http://

journals.openedition.org/hommesmigrations/1745; DOI : 10.4000/hommesmigrations.1745 


\section{Un corps en exil Entretien avec le danseur nigérian Qudus Onikeku}

Par Dagara Dakin, doctorant en histoire de l'art, université de Paris 1

Diplômé du Centre national des arts du cirque (Cnac) de Châlons-en-Champagne, en tant que danseur acrobate, Qudus Onikeku a été interprète pendant plus de dix ans sur la scène chorégraphique de la capitale nigériane, Lagos. II est, entre autres, à l'initiative d'un projet au titre

évocateur Do We Need Cola-Cola to Dance ? qu'il a mené au printemps 2008 avec une équipe composée d'un photographe, d'une danseuse et d'un vidéaste. Ce projet de danse dans la rue l'a conduit dans six villes

du continent africain. Interprète dans les créations de Heddy Maalem, notamment Le Sacre du printemps en 2009, il a tourné en Europe, aux États-Unis et au Brésil. De décembre 2009 à mai 2010, il était en résidence au 104 à Paris. II revient sur son approche de la notion d'exil, thème qui sous-tend son spectacle intitulé My Exile is in My Head, un projet qu'il a mené en collaboration avec le vidéaste Isaak Lartey, le guitariste Charles Amblard, l'éclairagiste Guillaume Fesneau et la photographe Agnès Chafei. 
"L'exil, s'il constitue étrangement un sujet de réflexion fascinant, est terrible à viore. C'est la fissure à jamais creusée entre l'être humain et sa terre natale, entre l'individu et son vrai foyer, et la tristesse qu'il implique n'est pas surmontable ${ }^{(1)}$."

\section{Dagara Dakin : Pouvez-vous nous raconter en quelques mots votre par- cours, comment vous êtes devenu danseur ?}

Qudus Onikeku : J'ai grandi en faisant des acrobaties, en voyant autour de moi les gens faire des "flips", j'ai surtout vu faire des flips arrière. Alors j'ai commencé à les imiter. Je faisais des séries de cinquante à la suite. Je le faisais surtout à l'extérieur, car au domicile familial c'était difficile. Je suis le dernier d'une famille de treize enfants et tous me sommaient de m'arrêter de peur que je ne me fasse mal. C'était leur façon de me protéger. J'étais le plus jeune et cela me frustrait car j'avais très envie de faire des acrobaties chez moi. Alors, à la moindre occasion, quand on m'envoyait faire des courses, sur le chemin de l'école, je m'exprimais à travers les acrobaties.

\section{D. : Quelle place a pris la danse dans votre scolarité ?}

Q. O. : À l'école, il y avait ce qu'on appelle les "clubs", des lieux d'expression en quelque sorte. Des membres du "club" dédié à la culture, "Cultural club", étaient venus dans ma classe et m'avaient proposé de les rejoindre. Ils recherchaient des individus qui aient un don artistique du type chant, danse, etc. Et lorsqu'ils m'ont parlé d'acrobatie, j'ai tout de suite souhaité participer. Au sein de ce "Cultural club", je me suis petit à petit intéressé à la danse et j'ai décidé de m'impliquer aussi dans cette discipline.

\section{D. : Vous avez donc très tôt fait le choix d'une filière artistique...}

Q. O.: J'ai été plutôt surpris, car étant inscrit à l'école dans une filière scientifique, j'avais l'impression qu'une discipline comme la danse n'était peut-être pas compatible avec ma filière d'origine. Mes parents pensaient d'ailleurs la même chose. Jusqu'à la fin du lycée, ils m'ont surtout demandé de me focaliser sur les études scientifiques. Je me suis donc beaucoup moins impliqué dans la danse que je considérais comme un divertissement. À 16 ans, j’ai quitté le lycée et là, j’ai pu reprendre la danse plus sérieusement. C'est à ce moment que j'ai décidé de devenir danseur professionnel.

\section{D.D. : À quand remonte votre premier séjour en France ?}

Q. O.: Je suis arrivé en France la première fois en 2004 pour intégrer la compagnie de danse de Heedy Maalem en qualité de danseur professionnel, également parce que j'étais anglophone puisque originaire du Nigeria. J'étais très jeune alors. 


\section{D.D. : Quelle idée vous faisiez-vous de la France avant votre arrivée ?}

Q. O.: Je n'avais aucune idée préconçue sur la France, je reliais peut-être ce pays au joueur de football "Jay-Jay" Okocha qui jouait alors au PSG ou à Zinedine Zidane, rien de plus. Le football était l'un des rares prismes au travers duquel nous pouvions percevoir de nombreux pays que nous ne connaissions pas. Mais si vous me parlez de l'Europe... Avant de venir en France, j'avais fait un bref séjour en Croatie, en Allemagne et au Royaume-Uni, notamment en 2001. Par conséquent, j’avais une idée de l'Europe.

\section{D. : Vous venez de clore une résidence de six mois au 104. Comment vous êtes-vous retrouvé dans ce lieu ? Pouvez-vous nous dire comment est né le projet My Exile is in My Head?}

Q. O.: Le 104 a fait un appel à projet et j’ai postulé. C'était en 2008, année d'ouverture de ce lieu culturel, je finissais mes études au Cnac. Je pensais à ce moment-là à traiter de la question des sans-abri et de la notion de "homeless", d'être sans domicile fixe, le fait d'être toujours un nomade, à la recherche d'un lieu. C'est de là qu'a émergé le projet.

Le titre m'est venu au cours des mois d'avril et mai 2009. Je pensais à cette chose que l'on nomme "home", la maison. Je me suis posé la question de savoir ce qu'était mon chez-moi. Et en pensant à mon enfance, au caractère polygame de la famille au sein de laquelle j’ai évolué, je me suis rendu compte que, très jeune, je cherchais déjà ma place.

\section{D. : Qu'est-ce que l'exil dont parle le titre de votre spectacle et comment} traduire ce concept dans la discipline qui est la vôtre?

Q. O.: L'exil que j'évoque n'a pas seulement à voir avec le fait de voyager. En réalité, il a plus trait à un questionnement sur soi, une recherche pour aller à la rencontre de soi, pour se créer selon l'image qui convient à soi-même, de définir ce que l'on veut pour soi-même.

L'exil est, selon moi, un passage obligé pour tout artiste. Cela correspond parfois à cette période de la vie où l'on commence à être incompris des gens qui nous entourent, alors commence parfois le genre d'exil dont je parle.

De nos jours, nous vivons dans une société qui nous oblige à nous positionner par rapport à tel ou tel courant politique, religieux, etc., à savoir dire à quel pays nous appartenons. Alors, j’ai moi-même ressenti le besoin de définir d'où je venais. Mais cette idée me perturbe, je n'aime pas l'idée de devoir appartenir à tel ou tel groupe, qu'il soit ethnique, religieux, politique, etc. 


\section{D. : Votre approche est donc très personnelle même si vous vous êtes nourri de la lecture d'auteurs tels que Wole Soyinka, Olu Oguibe ou encore Edward Said.}

Q. O.: Je ne suis pas à l'aise avec cette idée de définir le reste de l'humanité qui m'entoure comme "les autres". Voilà, pour moi l'idée de l'exil : c'est de n'appartenir réellement à aucun groupe. Mais attention, je ne souhaite pas que l'on assimile cela à une sorte de "crise existentielle" car ce n'est vraiment pas le cas ici. Je suis, disons, dans une dynamique qui va me permettre de porter attention à la moindre partie de ce qui fait mon "moi". Je suis nigérian, je suis yoruba, je suis africain, je suis hétérosexuel, je suis musulman, je suis danseur, je suis tout ça à la fois, aussi pour moi ça ne fait pas sens de ne m'attacher qu'à un seul aspect de ma personnalité. Je ne veux pas avoir à choisir un aspect au détriment des autres. Évidemment, à un moment donné on est obligé de choisir, par la force des choses, de se définir par rapport aux "autres". Je veux être dans la vie à part entière.

\section{D. : II n'est donc pas question d'un exil politique...}

Q. O. : Souvent le thème de l'exil est associé à une idée politique - évidemment, on ne peut pas nier cet aspect -, mais j'ai souhaité lui donner une autre dimension. En tant que danseur, j'avoue que j'éprouve une certaine difficulté à m'exprimer avec les mots. Mon corps est pour moi le meilleur outil d'expression. Via la danse, j'exprime ce que je suis. On pourra distinguer dans ma danse des éléments de hip-hop, de capoeira, de buto dancing, de danse contemporaine. Ce corps est ce que j'appelle un "corps incarné" parce qu'il s'alimente de différentes choses, différents styles pour finalement n'en faire qu'un.

\section{D. : La danse vous paraît donc être appropriée pour traiter ce thème ?}

Q. O.: Le thème de l'exil pourrait, d'après moi, se décliner en une multitude d'expressions artistiques autres que la danse, si je voulais aller plus loin dans la réflexion. Je pourrais utiliser l'écriture, la vidéo, la musique, les jeux de lumière... Mais je ne tiens pas à enfermer le public dans mon exil, je veux qu'il vienne me voir "performer" et qu'il se divertisse devant mon spectacle. Je ne pourrais pas dire dans un livre, tout ce que je pense sur la question de l'exil.

Cet entretien a été traduit de l'anglais vers le français avec l'aimable concours de Latifa Aït Naceur.

\section{Notes}

1. Edward W. Said, Réflexions sur l'exil et autres essais, Paris, Actes Sud, 2008, p. 241. 